Research Article

Zhenpeng Li* and Tang Xijin

\title{
Dynamics of Online Collective Attention as Hawkes Self-exciting Process
}

https://doi.org/10.1515/phys-2020-0002

Received Oct 29, 2019; accepted Dec 30, 2019

\begin{abstract}
Understanding the dynamic formation mechanism of online collective attention has been attracted diversified interests such as Internet memes, viral videos, or social media platforms and Web-based businesses, and has practical application in the area of marketing and advertising, propagation of information. Bulletin Board System, or BBS can be regarded as an ecosystem of digital resources connected and shaped by collective successive behaviors of users. Clicks and replies of the posts quantify the degree of collective attention. For example, the collective clicking behavior of users on BBS gives rise to the up and down of focus on posts, and transporting attention between topics, the ratio between clicks and replies measure the heat degree of a post. We analyzed the dynamics of collective attention millions of users on an interactive Tianya Zatan BBS. By analyzing the dynamics of clicks we uncovered a non-trivial Hawkes process self-exciting regularity concerning the impact of novelty exponential decay mechanism. Here, it able to explain the empirical data of BBS remarkably well, such as popular topics are observed in time frequently cluster, asymptotic normality of clicks. Our findings indicate that collective attention among large populations decays with a exponential decaying law, suggest the existence of a natural time scale over novelty fades. Importantly, we show that self-exciting point processes can be used for the purpose of collective attention modeling.
\end{abstract}

Keywords: Hawkes self-exciting process, collective attention, novelty decay, herding effect

\footnotetext{
^Corresponding Author: Zhenpeng Li: Dali University,Dali, China, 671003; Email: lizhenpeng@amss.ac.cn

Tang Xijin: Academy of Mathematics and Systems Sciences Chinese Academy of Sciences, Beijing, 100190, China; University of Chinese Academy of Sciences, Beijing, 100049, China; Email: xjtang@amss.ac.cn
}

Ә Open Access. ๑ 2020 Z. Li and T. Xijin, published by De Gruyter. (cc) BY 4.0 License

\section{Introduction}

From earthquake modelling to financial analysis, Hawkes process is an interesting class of stochastic model for 'selfexciting' processes. It is a counting process that models a sequence of 'arrivals' of some type over time, for example, financial markets collapse, earthquakes, trade orders, or hot topics searching on social media. Hawkes process depict a class of physical characteristics that each arrival excites the process in the sense that the chance of a subsequent arrival is increased for some time period after the initial arrival. As such, it is a non-Markovian extension of the Poisson process [1]. Figure 1 shows Baidu Index fluctuating trends of three major hot issues from December 2018 to September 2019. It is obvious that the index demonstrate in time frequently cluster, which suggest that the underlying process is indeed self exciting. In fact, the selfexciting in such case reflect human collective herding effect. The similar physic phenomenon are ubiquitous, such as an earthquake typically increases the following interval aftershocks [2], fighting between gangs is usually followed by a series of retaliations [3]. Hot-selling goods will lead to subsequent popularity, financial crisis through the world's financial centres will lead to continued worldwide financial market turbulence [4].

Obviously, the classic Poisson process is inappropriate to model such types of sequence of "arrivals" with time series clustering or burst characteristics. Similar investigation on indexing and pattern discovery in time series have also attracted huge interest [5-8], but it worth to note that none of these methods specifically focused on modeling bursts.

\section{Collective attention and clicks}

Every second, 600,000 messages are shared on Facebook across the Internet, 200 million emails, 100,000 tweets are sent, and 571 new websites are created [9]. As Herbert Simon, the winner of the economics prize, pointed out "in an information rich world, having information means an- 


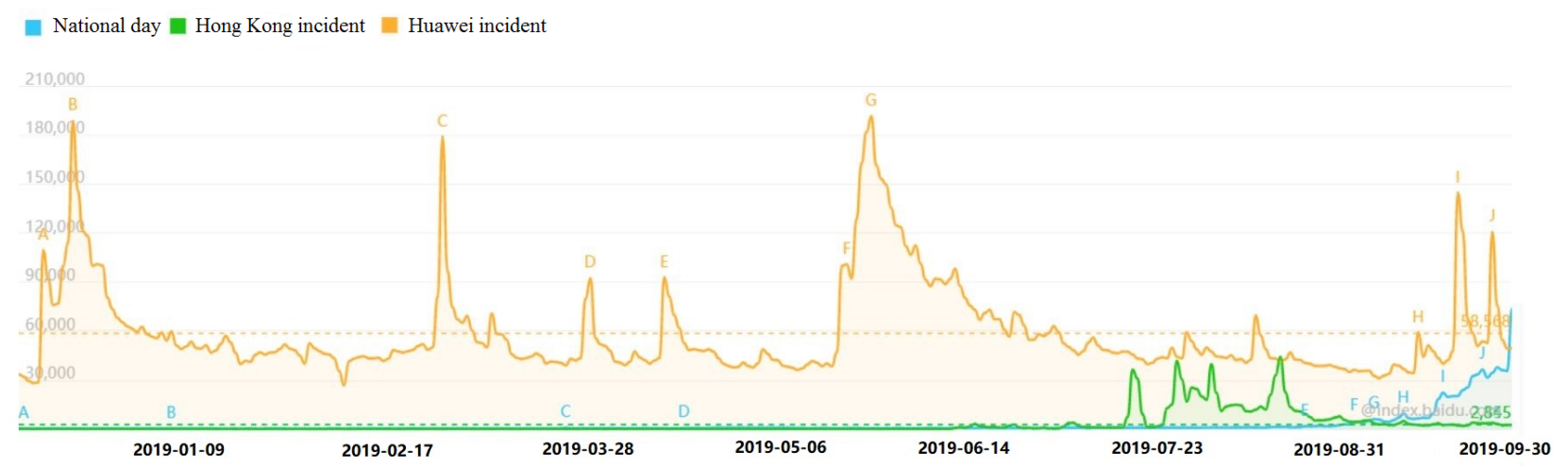

Figure 1: Baidu index fluctuation (from 2018-12-01 to 2019-9-30) of three hot key words (National day, Hong Kong incident, Huawei incident).

other scarce resource", which is the attention of information receivers [10]. Because of the importance and scarcity of attention [10], M. Goldharbor et al. first developed the concept of attention economy, and the qualitative study of attention [11]. However, the quantitative study of human group attention did not really begin until the 1990s.

Collective attention is firstly presented by B. Huberman. It refers to the attention and access of a large number of groups to limited information resources, including clicks, comments, modifications and so on [12].

In recent years, the in-depth development of social media has prompted scholars to study the competition of multiple information resources for limited attention [13]. This competition can be simplified as a critical branching process and contains a critical phenomenon [14]. On the other hand, collective attention also shows the characteristics of burst in time, especially before and after the arrival of hot events, there will be different outbreak patterns $[15,16]$.

Collective attention both exhibits abundant temporal and spatial dynamics, and plays important role on knowledge creation [17, 18], stock fluctuation prediction [19], political prediction [20], scientists interest transfer [21], extreme event prediction [22], etc.

Clicks reflect the important online users surfing behavior. The approach of clicks analysis provides a window to observe collective online activities. For example, clicks analysis can help us to understand the allocation and transmission of users' attention among news, present us a approach to explain the rise and decay of topics from the aspect of users' novelty decay [12]. In previous studies, the click stream are also used to measure the collective direction of attention diffusion [23], or human knowledge maps [24]. These pilot empirical studies show that clicks can be used to measure online group attention dynamic.

To understand the process underlying attention on BBS, consider as an example how a new post spreads among a group of people. After it first published, the topic attract the attention of a few, then the clicks may increase constantly if they find it interesting enough. With people continuously paying attention to the post, more people may visit the post, and clicks will continue to increase. That means a positive-reinforcement effect or herding effect sets in such that trigger a short-term surge in clicks. After a couple of hours or days its cumulative clicks increasing rate slows down, even remains stagnant because of both its lack of novelty and its lack of prominent visibility (it was covered up by new posts). Thus, the cumulative clicks of each post eventually saturates to a value $N(t), t \longrightarrow \infty$ that depends on both its popularity and its novelty decay. Collective clicking behavior in this example exhibit a herding effect, displaying properties that characterize self-exciting Hawkes process.

In the following, based on our empirical observation that strong cluster pattern in clicking time series in Tianya Zatan, we derive the general mechanism that reflect the underling attention dynamics of rapid initial growth and prolonged decline. We discuss the stochastic mathematical model - Hawks process, its application on collective clicks, and provide plausible explanations as to quantify the degree of collective attention.

In the next section, firstly, we briefly give definitions for counting processes. Secondly, we discuss the exponential decaying conditional intensity function, and its associated Hawks counting process.

\section{Counting and point process}

In order to use Hawkes process to describe collective online clicking behaviors to measure the collective online attention, before turning to the application of Hawkes pro- 
cess for collective clicking modeling, some core concepts for a clear understanding of Hawkes process are given in this section.

Definition 1 (Counting process). A stochastic process $\{N(t), t \leq 0\}$ is a counting process, where $N(t)$ taking values in natural number set, satisfies $N(0)=0$, is almost surely finite. $N(t)$ is a right-continuous step function with increments of size plus 1 . We regard a counting process $N(t)$ as a cumulative count of the number of "arrivals" into a system up to the current time. Consider the sequence of users' clicking random arrival times $T=\left\{t_{1}, t_{2}, \ldots\right\}$, at each $t_{j}, N\left(t_{j}\right)$ has a jump. Each clicking behavior excites the process in the sense that the chance of a subsequent clicking arrival is increased for some time period after the initial clicking arrival, therefore collective clicking amount $\{N(t), t \geq 0\}$ is a counting process. The underlying process is indeed self exciting, because online participants prefer to exhibit a herding behaviour [25]. This behaviour causes temporal clustering of users' clicking random arrival times $T=\left\{t_{1}, t_{2}, \ldots\right\}$. That means using the Poisson process to model the arrival of clicks of posts is highly inappropriate. The counting process defined as these arrival times is also named a point process, if $T=\left\{t_{1}, t_{2}, \ldots\right\}$ satisfy $P\left(0 \leq t_{1} \leq t_{2} \leq \ldots\right)=1$, and the number of points is almost surely finite [26].

Definition 2 (Conditional intensity function). Consider a counting process $N(t)$ with associated history $\sigma(\cdot)$, if $\lambda^{*}(t)$ exists such that $\lambda^{\star}(t)=\lim _{h \infty} \frac{E(N(t+h)-N(t) \mid \sigma(\cdot))}{h}$ which only relies on the historic observation of $N(\cdot)$, i.e. $\lambda^{\star}(t)$ is $\sigma(\cdot)$ measurable, then $\lambda^{*}(t)$ is named the conditional intensity function of $N(t)$.

Definition 3 (Hawkes process). A counting process $N(t)$ : $t \geq 0$ with associated history $\sigma(t): t \geq 0$, that satisfies

$$
P(N(t+h)-N(t)=m \mid \sigma(t))=\left\{\begin{array}{l}
\lambda^{\star}(t) h+o(h), m=1 \\
o(h), m>1 . \\
1-\lambda^{\star}(t) h+o(h), m=0
\end{array}\right.
$$

Such a process $N(t)$ is a Hawkes process. Where the Hawkes process conditional intensity function takes the form

$$
\lambda^{\star}(t)=\lambda_{0}+\int_{0}^{t} \mu(t-u) d N(u)
$$

for some background intensity $\lambda_{0}>0$ and excitation function $\mu:(0, \infty) \longrightarrow[0, \infty]$. In the trivial case of $\mu(\cdot)=0$ the Hawkes process turns into classic homogeneous Poisson process with mean $\lambda_{0} h$, in other word, $P(N(t+h)-N(t)=$ $m \mid \sigma(t))=P(N(t+h)-N(t)=m)=\frac{\left(\lambda_{0} h\right)^{m}}{m !} e^{-\lambda_{0} h}$.
In section 4, we use self-exciting Hawkes process (the collective clicks) to measure the degree of collective attention. The excitation function play pivotal role in describing the online group self exciting process. Based on our empirical observation, we find that the collective novelty decay pattern on Tianya Zatan is an exponential decay as shown in Figure 2. For the reason, the remaining discussion will focus on the exponential form of the self excitation function, i.e., $\mu(t)=\alpha e^{-\beta t}$ with two constant parameters $\alpha, \beta>0$. The two constant parameters have the following interpretation: self excitation function $\mu(t)$ increases with $\alpha$, and reduces along with the $\beta$ increases. Next we focus the case of Hawkes process with exponentially decaying exciting function.

Consider the characteristics of Hawkes process asymptotic normality [27], i.e., $d N(u)=\frac{1}{\sqrt{2 \pi}} e^{\frac{u^{2}}{2}} d u$, and the case of Hawkes process with exponentially decaying exciting function, a natural extension of Eq. (1) gives

$$
\lambda^{\star}(t)=\lambda_{0}+\int_{0}^{t} \alpha e^{-\beta(t-u)} \frac{1}{\sqrt{2 \pi}} e^{\frac{-u^{2}}{2}} d u
$$

Applying calculus to Eq. (2) with upper limit $T$ we have

$$
\begin{aligned}
N(T) & =\int_{0}^{T} \lambda^{\star}(t) d t \\
& =\int_{0}^{T} \lambda_{0} d t+\int_{0}^{T}\left(\int_{0}^{t} \alpha e^{-\beta(t-u)} \frac{1}{\sqrt{2 \pi}} e^{\frac{-u^{2}}{2}} d u\right) d t \\
& =\int_{0}^{T} \lambda_{0} d t+\int_{0}^{T}\left(\frac{\alpha}{\sqrt{2 \pi}} e^{\frac{\beta^{2}}{2}-\beta t} \int_{0}^{t} e^{\frac{-(u-\beta)^{2}}{2}} d u\right) d t \\
& =\int_{0}^{T} \lambda_{0} d t+\int_{0}^{T} \alpha e^{\frac{\beta^{2}}{2}-\beta t}(\Phi(t)-0.5) d t \\
& \leq \int_{0}^{T} \lambda_{0} d t+\frac{1}{2} \int_{0}^{T} \alpha e^{\frac{\beta^{2}}{2}-\beta t} d t \\
& =\lambda_{0} T+\frac{1}{2} e^{\frac{\beta^{2}}{2}} \frac{\alpha}{\beta}\left(1-\frac{1}{e^{\beta T}}\right) \\
& \leq \lambda_{0} T+\frac{1}{2} e^{\frac{\beta^{2}}{2}} \frac{\alpha}{\beta} .
\end{aligned}
$$

Where $\Phi(\cdot)$ is the cumulative distribution function of the standard normal distribution. Eq. (3) suggest that for finite time scale $T$, Hawkes process $(N(t): t \geq 0$ ) has upper bound, meanwhile increases linearly with time $t$. 


\section{Modelling and empirical analysis of clicks}

To test the performance and robustness of our model, we consider the Tianya Zatan dataset.

\subsection{Datasets}

Founded in 1999, as a famous Chinese BBS, Tianya (or Tianya club) is the leading public BBS social media platform (http://bbs.tianya.cn/). It advocates self-attention, social concern, humanity concern, and plays the role of leading real world public opinion, with rapid sensitive response and distinctive position. We obtained 1,241,674 posts published in Tianya Zatan board during the time span from 2012/1/1 00:00:00 to 2015/12/31 23:59:00. The information of each post includes: title, author, the initial posting time, reply time stamps, clicking time stamps, clicking volume, and replies. Clicking on the title of the post one can access more detailed information. The main page of Tianya Zatan board contains 80 posts, which are sorted according to the time reversal. Only the latest posts published or the latest replies will appear in the front of the page. Most old posts or unpopular posts were covered by new or popular posts.

\subsection{Collective novelty decaying on Tianya Zatan}

In this paper, for all posts, we obtain its published time and the first reply time to the post. Then we use the probabilistic density distribution (pdf) of first-reply time interval to a new post in the first 120 mins as an index to measure collective novelty decay on Tianya Zatan. The result suggests that more than $28 \%$ posts obtain the first reply in 5 minutes. The collective novelty decay on Tianya Zatan indeed exponential decay as shown in Figure 2. We calculate the first-reply time interval to a new post, such as $[0,5],[5,10], \ldots,[105,120]$, time unit is minute, and plot Figure 2. The abscissa in this figure is the average value of each time interval (in log scale), $\times$ represents replying probabilistic density to a new post corresponding to the time interval. The result is consistent with the empirical finding that decay in novelty can be fit to a stretched exponential relaxation law in digg.com [12].

Here we use the collective clicks to measure the degree of collective attention. The novelty decay function becomes the key underlining dynamic mechanism to de-

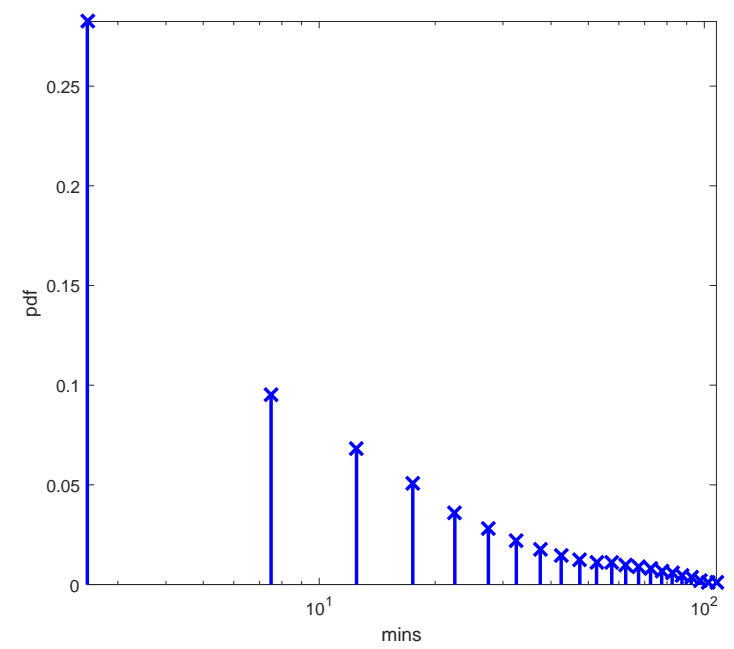

Figure 2: Novelty decay on Tianya Zatan in the first 120 mins.

scribe the online group self-exciting process. For the reason, the remaining discussion will focus on the exponential form of the excitation function, i.e., $\mu(t)=\alpha e^{-\beta t}$ with two constant parameters $\alpha, \beta>0$. The two constant parameters have the following interpretation: each click arrival in the system

instantaneously increases the arrival intensity by $\alpha$, then over time this arrival's influence decays at rate $\beta$.

\subsection{Distribution of collective clicks}

To determine the statistical distribution of collective clicks $N(t)$ on Tianya Zatan, when $t \longrightarrow \infty$ in mathematical definition, reflected in the fact that $t$ is large enough, for example a couple of months or years. Which corresponds to the number of each post clicks that accumulated throughout forum evolution. We plot the histogram of all posts clicks from 2012/1/1 00:00:00 to 2015/12/31 23:59:00. As shown in Figure 3, the clicks follows normal distribution with mean 2.513 and standard deviation 0.775. A KolmogorovSmirnov normality test yields a p-value $<1.8^{-10}$ and testing statistics $D=0.076$. Small $D$ suggests that collective clicks follow a normal distribution. Both Quantile-Quantile test and the obvious statistic result are well matched with the characteristics of Hawkes process asymptotic normality with the exception of the high and low end of the distributions. Meanwhile it directly proof that Eq. (3) is reasonable. 


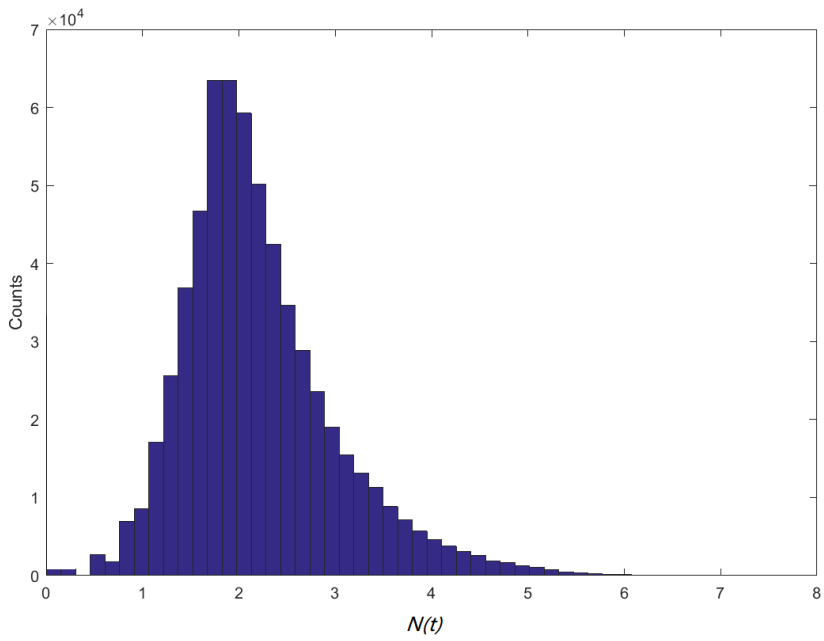

Figure 3: Clicking volume distribution of 4 years in Tianya Zatan.

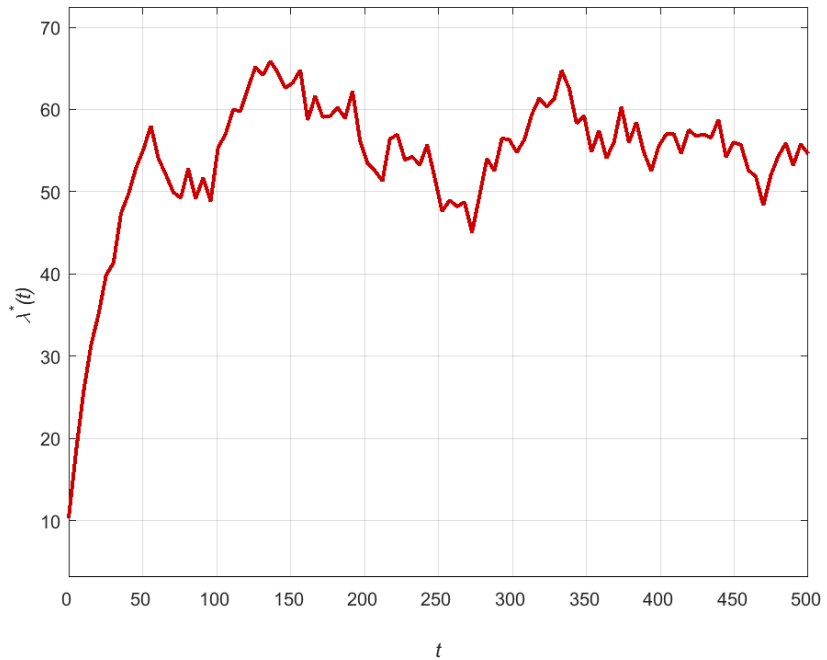

Figure 4: Condition density function of an Hawks process with parameters $\lambda_{0}=10, \alpha=0.1734, \beta=0.2133$.

\subsection{Modeling collective clicks}

We use the average arrival times of clicking process for the all 4 years posts as empirical observation data, and apply Hawkes process likelihood estimation method provided in [28], obtain background density $\lambda_{0}=10$, density enhanced parameter $\alpha=0.1734$, arrival influence decaying parameter $\beta=0.2133$.

Consider the case of Hawkes process with exponentially decaying exciting function as in Eq. (2), we simulated the Hawkes process with parameters $\lambda_{0}=10, \alpha=0.1734$, $\beta=0.2133$. Figure 4 shows that a spike at short time, and indicates an increased likelihood of another clicking following each clicking. This is a typical burst phenomenon in human dynamics. Self-exciting induce fluctuation of collective attention, reflecting condition density function

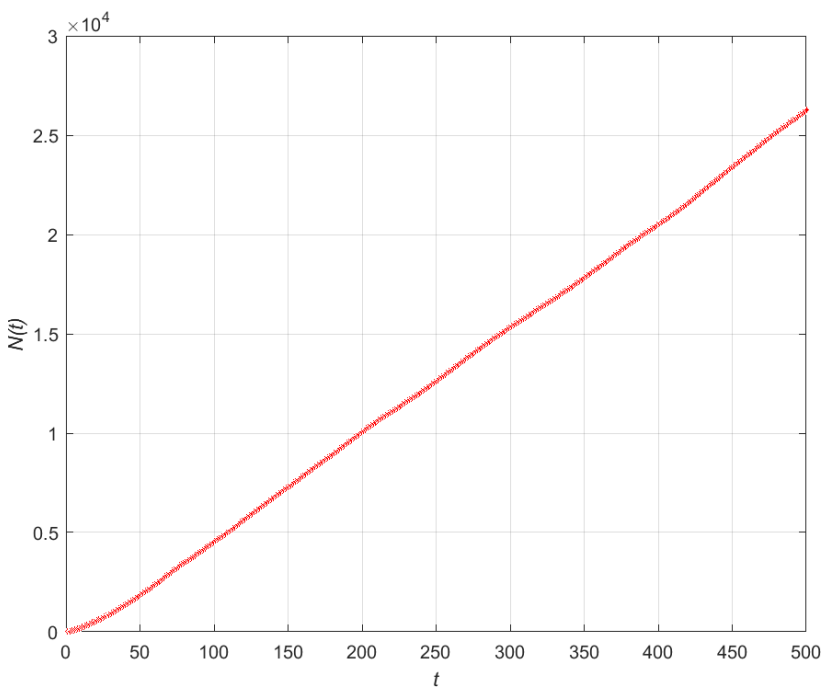

Figure 5: An Hawks process based clicking volume dynamic growth with parameters $\lambda_{0}=10, \alpha=0.1734, \beta=0.2133$.

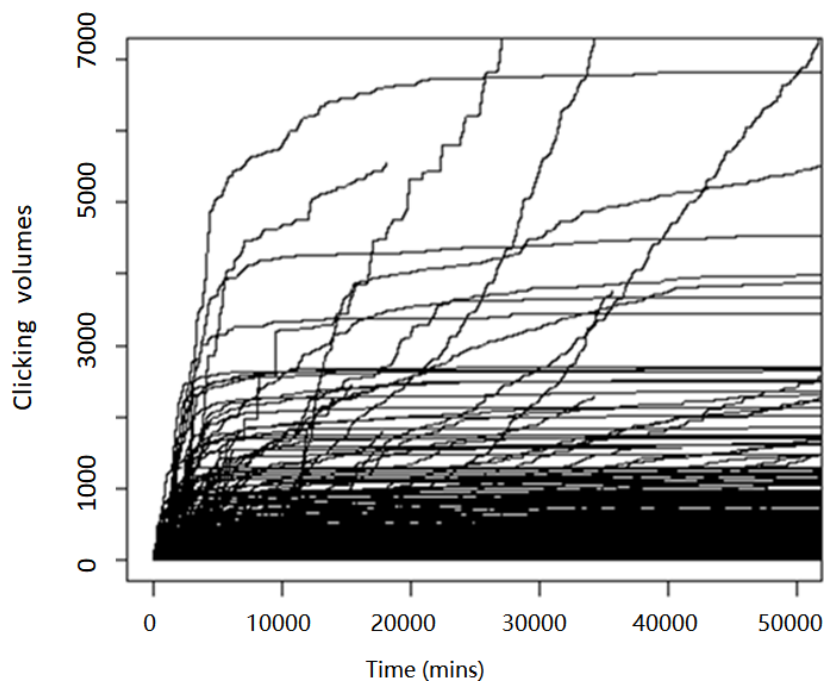

Figure 6: Clicks of posts increase linearly with time before novelty decreases over time

variation. For example, as respect to online collective attention, for a hot topic, we see that both Figure 1 and Figure 4 experience three stages: Imitation, Saturation, Competition . This suggests that the ups and downs of collective attention, is driven by increasing production and consumption of topics (contents), and the interplay with competition for topics' novelty, resulting in a more rapid exhaustion of limited attention resources. Meanwhile, we observe that Hawkes process $(N(t): t \geq 0)$ increases linearly with time $t$, then consecutively tends to a stable level as illustrated in Figure 5 and Figure 6. The result is consistent with our theoretic analysis in Section 3. 
Table 1: The basic statistic of the selected 6 posts

\begin{tabular}{|c|c|c|c|c|}
\hline $\begin{array}{l}\text { topic } \\
\text { and } \\
\text { URL }\end{array}$ & $\begin{array}{l}\text { publish } \\
\text { time }\end{array}$ & $\begin{array}{l}\text { total } \\
\text { clicking } \\
\text { counts }\end{array}$ & $\begin{array}{l}\text { total } \\
\text { replying } \\
\text { counts }\end{array}$ & $\begin{array}{l}\text { last } \\
\text { replying } \\
\text { time }\end{array}$ \\
\hline (Understanding after 40: harmonious health and less & $2012 / 01 / 01$ & 44344 & 1029 & $2013 / 10 / 28$ \\
\hline illness) & $12: 49: 00$ & & & $18: 32: 00$ \\
\hline \multicolumn{5}{|l|}{ http://bbs.tianya.cn/post-free-2352187-1.shtml } \\
\hline (Why don't the Chinese abolish the custom of eating & $2012 / 1 / 1$ & 9678 & 147 & $2016 / 4 / 17$ \\
\hline together?) & $20: 24: 00$ & & & 23:17:00 \\
\hline \multicolumn{5}{|l|}{ http://bbs.tianya.cn/post-free-2352370-1.shtml } \\
\hline (Not understanding and sorrow for Chinese military) & $2012 / 1 / 1$ & 2853 & 100 & $2013 / 4 / 1$ \\
\hline http://bbs.tianya.cn/post-free-2352392-1.shtml & $21: 24: 00$ & & & $11: 24: 00$ \\
\hline \multirow[t]{2}{*}{ (Shouguang is in pain...) } & $2012-01-02$ & 1091 & 44 & $2013 / 2 / 10$ \\
\hline & $15: 47: 00$ & & & $14: 31: 00$ \\
\hline \multirow[t]{2}{*}{ (Tianya observation 491: 2012, Let’s paddle) } & $2012-01-03$ & 6295 & 393 & $2012-12-28$ \\
\hline & $14: 02: 00$ & & & $20: 22: 59$ \\
\hline \multirow[t]{2}{*}{ (2012 nature and culture prediction) } & $2012-01-06$ & 3261 & 251 & $2016-11-21$ \\
\hline & $17: 32: 00$ & & & $10: 22: 13$ \\
\hline
\end{tabular}

Then we plot the cumulative clicking counts for 5394 new posts of TianYa Zatan (selected from 1/1/2012 0:00 to 3/31/2012 23:57, the basic statistic of the selected 6 posts is shown in Table 1.) as a function of time steps (mins) in Figure 6. It is apparent that each post cumulative clicking volume shows an approximate initial linear growth, and then consecutively tends to a stable level. This trend is due to the reason that interest of everyone is very high at the beginning of the posts were published, then the novelty decreases exponentially over time. Comparing simulation result in Figure 5 with empirical plot in Figure 6, we propose that self-exciting point processes can be adapted for the purpose of collective attention modeling and are well suited to capture the temporal clustering patterns observed in TianYa Zatan.

\subsection{Immigration-birth analysis of clicks}

Respect to collective clicking behavior to new published posts and old ones, we regard Hawkes process $N(t)$ as a branching process. Hawkes process imagine counting the clicks in Tianya Zatan, where clicks either on new published posts or on old ones. Specifically to say, at time stamp $t$, Hawkes process $N(t)$ is composed of two parts,
$N(t)=N_{c}(t)+N_{i}(t)$. Where $N_{i}(t)$ denotes the count of clicks on new posts, while $N_{c}(t)$ indicates the count of clicks on old posts. $n=\int_{0}^{\infty} \alpha e^{-\beta t} d=\frac{\alpha}{\beta}$ is known as branching ratio in the case of exponentially decaying intensity. A counting process $(N(t): t \geq 0)$ explosion i.e., $N(t) \longrightarrow \infty$ as $t \longrightarrow \infty$ is avoided by ensuring that $\alpha<\beta$. When the branching ratio $0<n<1$, it can be interpreted as a probability. Therefore, any clicking arrival selected at random was generated endogenously (a child), i.e. clicking on old posts with probability $n$, or exogenously (an immigrant), i.e., clicking on new posts with probability $1-n$. An illustration of this interpretation is shown in Figure 7. A numerical calculation gives $\hat{\alpha}=0.1734, \hat{\beta}=0.2133$, and immediately we have $\hat{n}=0.8129$. This means that for each clicking arrival, there are over $80 \%$ chance that it belongs to $N_{c}(t)$. 


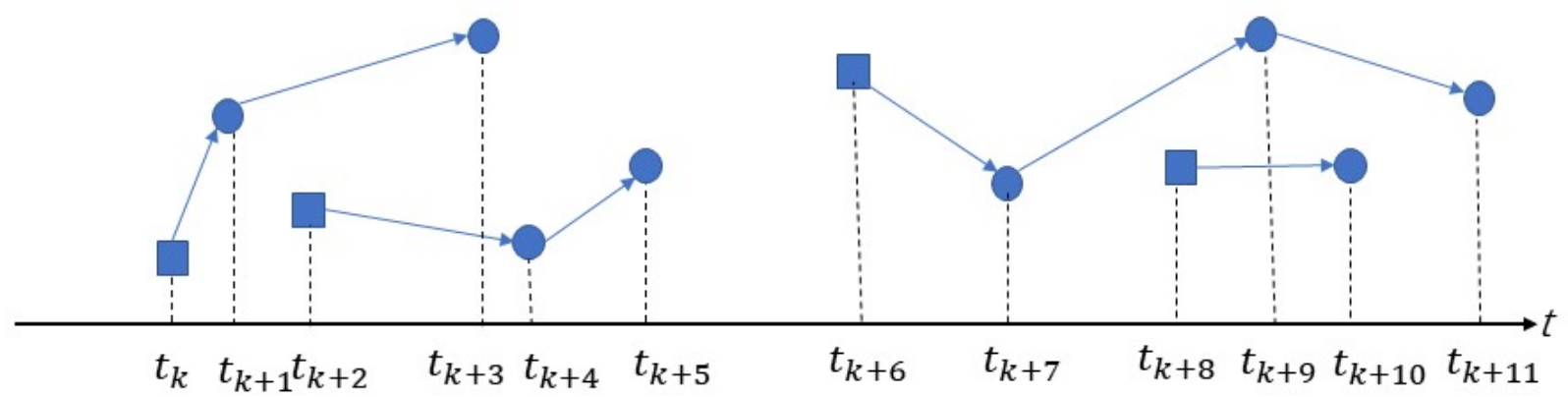

Figure 7: Collective clicking Hawkes process represented as a immigration-birth branching point process. Squares indicate clicking on new posts, circles denote clicking on old posts.

\section{Conclusion}

In this paper, we showed that the growth and decay of collective attention can be measured with dynamics of clicks and modeled by Hawkes process with exponentially decaying exciting function. The function includes two important parameters $\alpha, \beta$. Each click arrival instantaneously increases the arrival intensity by $\alpha$, then over time this arrival's influence decays at rate $\beta$. More specifically, parameter $\beta$ determines the natural time scale over which attention fades, and parameter $\alpha$ depicts herding effect, which manifests as temporal clustering of collective attention. In conclusion, through 4 years Tianya Zatan empirical data, we observe strong regularities of collective clicks in time series that reflect attention dynamics, that provide plausible explanations as to what drives the apparently dominant dynamics of rapid initial growth and prolonged decline of collective clicking behavior.

The investigation in this study can be implemented for social medias applications. For example, The principle of self-exciting process can be applied in designing the optimal dynamic Web system, can be use to boost Web-based businesses, other practical applications include online advertising, e-marketing and online risk management and control. For example, as respect to online public opinion regulation, based on the novelty competition and human limited attention mechanism, in order to resolve the online collective behavior, and then control the further spreading of online public opinion, we might adopt the novel topic transfer strategy, i.e., release enough novel contents at the Imitation step. For future possible work, we will consider the temporal features of clicking behavior along with other driving factors such as aging, freshness of topic, sentiment of title and social network structure etc, with the aim to describe collective online behaviors more precisely.
Acknowledgement: This research was supported by the National Natural Science Foundation of China under the grant Nos 71661001, 61473284, 71731002 and 71971190.

\section{References}

[1] Hawkes AG. Spectra of some self-exciting and mutually exciting point processes. Biometrika. 1971;58(1):83-90.

[2] Ogata Y. Statistical models for earthquake occurrences and residual analysis for point processes [J]. J Am Stat Assoc. 1988;83(401):9-27.

[3] Mohler GO, Short MB, Brantingham PJ, Schoenberg FP, Tita GE. Self-exciting point process modeling of crime [J]. J Am Stat Assoc. 2011;106(493):100-8.

[4] Azizpour S, Giesecke K, Schwenkler G. Exploring the sources of default clustering [J]. J Financ Econ. 2018;129(1):154-83.

[5] Faloutsos C, Ranganathan M, Manolopoulos Y. Fast subsequence matching in time-series databases [M]ACM; 1994. https://doi.org/10.1145/191839.191925.

[6] J, Keogh E, Lonardi S, et al. Visually mining and monitoring massive time series[C]//Proceedings of the tenth ACM SIGKDD international conference on Knowledge discovery and data mining. ACM, 2004: 460-469.

[7] Papapetrou P, Athitsos V, Potamias M, et al. Embedding-based subsequence matching in time-series databases[]]. ACM Transactions on Database Systems (TODS), 2011, 36(3): 17.

[8] Sakurai Y, Papadimitriou S, Faloutsos C. Braid: Stream mining through group lag correlations[C]//Proceedings of the 2005 ACM SIGMOD international conference on Management of data. ACM, 2005: 599-610.

[9] Website of Internet live Stats, available from http://www. internetlivestats.com/

[10] Simon HA. Designing Organizations for an Information-Rich World. Martin Greenberger, Computers, Communication, and the Public Interest. Baltimore (MD): The Johns Hopkins Press; 1971.

[11] Lei Z. Research on the attention economy school in the west. China Social Sciences Press; 2009.

[12] Wu F, Huberman BA. Novelty and collective attention. Proc Natl Acad Sci USA. 2007 Nov;104(45):17599-601.

[13] Weng L, Flammini A, Vespignani A, Menczer F. Competition among memes in a world with limited attention. Sci Rep. 
2012;2(1):335.

[14] Gleeson JP, Ward JA, O'Sullivan KP, Lee WT. Competition-induced criticality in a model of meme popularity. Phys Rev Lett. 2014 Jan;112(4):048701.

[15] Lehmann J, Gonçalves B, Ramasco JJ, et al. Dynamical classes of collective attention in twitter, Proceedings of the 21st international conference on World Wide Web. ACM, 2012: 251-260.

[16] Crane R, Sornette D. Robust dynamic classes revealed by measuring the response function of a social system. Proc Natl Acad Sci USA. 2008 Oct;105(41):15649-53.

[17] Ciampaglia GL, Flammini A, Menczer F. The production of information in the attention economy. Sci Rep. 2015 May;5(1):9452.

[18] Wu L, Baggio J, Janssen MA. The Dynamics of Collaborative Knowledge Production. arXiv preprint arXiv:1509.05083, 2015.

[19] Heiberger RH. Collective attention and stock prices: evidence from Google Trends data on Standard and Poor's 100. PLoS One. 2015 Aug;10(8):e0135311.

[20] Eom YH, Puliga M, Smailović ], Mozetič I, Caldarelli G. Twitterbased analysis of the dynamics of collective attention to political parties. PLoS One. 2015 Jul;10(7):e0131184.
[21] Huberman B A. Social Attention in the Age of the Web. Working together or apart: Promoting the next generation of digital scholarship, 2009, 62.

[22] Miotto JM, Altmann EG. Predictability of extreme events in social media. PLoS One. 2014 Nov;9(11):e111506.

[23] Wang CJ, Wu L, Zhang J, Janssen MA. The collective direction of attention diffusion. Sci Rep. 2016 Sep;6(1):34059.

[24] Davenport TH, Harris JG, Kohli AK. How do they know their customers so well? MIT Sloan Manag Rev. 2001;42(2):63.

[25] Onnela JP, Reed-Tsochas F. Spontaneous emergence of social influence in online systems [J]. Proc Natl Acad Sci USA. 2010 Oct;107(43):18375-80.

[26] Carstensen L. Hawkes processes and combinatorial transcriptional regulation [D]University of Copenhagen; 1910.

[27] Hawkes AG, Oakes D. A cluster process representation of a selfexciting process. J Appl Probab. 1974;11(3):493-503.

[28] Daley D, Vere-Jones D. An Introduction to the Theory of Point Processes: Volume I: Elementary Theory and Methods (Springer, 2003) 